\title{
Anabases
}

ANABASES Traditions et réceptions de l'Antiquité

$15 \mid 2012$

Varia

Ne pas quitter « la terre d'Afrique ». Les

Antiquisants, l'Algérie française et la déclaration du 23 mai 1956

Sarah Rey

(2) OpenEdition

Journals

Édition électronique

URL : http://journals.openedition.org/anabases/3689

DOI : $10.4000 /$ anabases.3689

ISSN : 2256-9421

Éditeur

E.R.A.S.M.E.

Édition imprimée

Date de publication : 1 avril 2012

Pagination : 71-84

ISSN : $1774-4296$

Référence électronique

Sarah Rey, « Ne pas quitter «la terre d'Afrique ». Les Antiquisants, I'Algérie française et la déclaration du 23 mai 1956 », Anabases [En ligne], 15 | 2012, mis en ligne le 01 avril 2015, consulté le 21 octobre 2019. URL : http://journals.openedition.org/anabases/3689 ; DOI : 10.4000/anabases.3689

(c) Anabases 
Anabases 15 (2012), p. 71-84

\section{Ne pas quitter " la terre d'Afrique ». Les Antiquisants, l'Algérie française et la déclaration du 23 mai 1956}

SARAH REY

$L_{E}$ MONDE DU 23 maI 1956 publie la déclaration de vingt-six professeurs de la Sorbonne, " exprimant leur adhésion réfléchie à l'effort militaire qui est demandé au pays et approuvant le récent appel "pour le salut et le renouveau de l'Algérie française " ". Les signataires rajoutent qu' "ils réprouvent l'injustice qu'il y aurait à renier, par ignorance ou par passion, les bienfaits d'une ouvre dont aucun esprit honnête ne met en doute la valeur. Cette œuvre est de celles dont les Français doivent rester fiers. Elle doit être continuée ». Ce texte se poursuit par un témoignage de solidarité à l'égard des " collègues d'Alger ", c'est-à-dire les enseignants de l'université de cette ville. Parmi les noms figurant en tête de cette déclaration, celui de Raymond Aron interpelle immédiatement le lecteur d'aujourd'hui. Mais, en entrant dans le détail de cette liste, il est intéressant d'observer la forte proportion de professeurs d'histoire grecque et romaine : Jean Bérard, André Boulanger, Pierre Boyancé, Pierre Demargne, Jacques Heurgon, Charles Picard, Jacques Perret, William Seston et Pierre Wuilleumier. Comment peut-on expliquer cette forte mobilisation du camp antiquisant?

Ces intellectuels n'ont pas dû supporter qu'un des leurs, Henri-Irénée Marrou, publie, au début d'avril 1956, dans le même quotidien, une tribune retentissante intitulée "France, ma patrie ${ }^{2}$ " où sont âprement décrits certains agissements de militaires français et les dérives du conflit algérien. Marrou y évoque " des laboratoires de torture » et va jusqu'à comparer aux pratiques nazies les violences exercées contre les

$1 \quad$ Le Monde, 23 mai 1956.

2 Le Monde, 5 avril 1956. 
Algériens et ceux qui soutiennent leur émancipation. Entre les lignes, le texte du 23 mai passe donc pour une réponse masquée au franc-tireur Marrou.

Celui-ci a osé se jeter dans la mêlée de la guerre d'Algérie et dans ses corollaires de papier, c'est-à-dire les multiples pétitions, lettres ouvertes, manifestes, pamphlets qui, dans la presse et l'édition françaises, jalonnent le conflit. Les antiquisants ont eu leur part dans cette "bataille de l'écrit » (déjà amplement analysée) débutant dès 1955 et se poursuivant jusqu'en $1962^{3}$. Ils sont ces " chers professeurs " tournés en dérision par Maurice Bourgès Maunoury, ministre de la défense du gouvernement de Guy Mollet : ces clercs de la République solidement installés dans leur rôle social ${ }^{4}$.

Pourquoi ces individus tiennent-ils à rendre public leur point de vue sur les événements algériens ? Sont-ils d'ardents colonialistes, dépassés par l'actualité ? Le conservatisme est-il inscrit dans le code génétique des professeurs de la Sorbonne ? À l'exception d'Aron, dont l'activité politique est déjà connue à cette époque, les signataires de la déclaration de mai 1956 semblent s'être forcés à sortir de leur traditionnelle réserve dans un contexte tendu. En effet, le gouvernement de Guy Mollet, en mars de cette même année, s'est vu octroyer " des pouvoirs spéciaux ", qui lui donnent, ainsi qu'au ministre résident en Algérie, Robert Lacoste, une immense marge de manœuvre, aux frontières de la légalité ${ }^{5}$. Le FLN multiplie ses attentats contre les civils. Le 18 mai 1956, une embuscade dans le défilé de Palestro fait vingt morts chez les rappelés du $9^{\text {e }}$ RIC $^{6}$. Le 19 mai, lycéens et étudiants entament, à Alger, une grève à l'appel du FLN ${ }^{7}$. Le 23 mai enfin, en désaccord avec la politique algérienne du gouvernement, le ministre Pierre Mendès France démissionne.

Dans cette période de grands troubles, il est difficile de situer politiquement ces professeurs de la Sorbonne, auteurs d'une profession de foi dans l'Algérie française. Ces hommes appartiennent, en majorité, à la même génération : beaucoup sont nés entre 1900 et 1908, ils ont autour de cinquante ans au moment des faits, hormis André Boulanger et Charles Picard qui sont alors septuagénaires. Tous mènent une

3 Cf. J.-P. Rioux \& J.-Fr. Sirinelli (dir.), La guerre d'Algérie et les intellectuels français, Paris, Éditions Complexe, 1991, p. 22. La formule est contestée par Jean-Marie DomenaCH : "Le seul point sur lequel je sois en désaccord complet avec les rapporteurs est l'expression "la guerre de l'écrit". [Pendant la guerre] J'ai passé beaucoup plus de temps à parler, et surtout à agir : faire des réunions, constituer des comités, organiser des "actions", faire des voyages" (ibid., p. 354).

$4 \quad$ Ils se placent dans le sillage d'un autre professeur passé à la politique, Jacques Soustelle (1912-1990), fondateur de "l'Union pour le salut et le renouveau de la France " et gouverneur général d'Algérie au début du conflit. Soustelle a été le condisciple de Jean Bérard rue d'Ulm (promotion 1929).

5 De l'autre côté de la Méditerranée, le ministre résident, Robert Lacoste, peut « instituer des zones où le séjour des personnes est réglementé ou interdit, avec la possibilité de déléguer ce pouvoir aux préfets et aux autorités militaires ".

6 R. Branche, L'embuscade de Palestro. Algérie 1956, Paris, Armand Colin, 2010, 256 p.

7 D'où la mention, dans la déclaration du 23 mai, des « collègues » d'Alger. 
vie publique discrète et, bien souvent, rien ne transparaît dans leurs œuvres de leurs opinions politiques.

Au-delà de la riposte à Marrou, les antiquisants signataires de la déclaration du 23 mai se sont mobilisés en souvenir de leur expérience africaine. Parmi eux, nombreux sont ceux qui, dans leur jeunesse, ont en effet pratiqué l'archéologie dans les pays d'Afrique du Nord. Depuis le XIX ${ }^{\mathrm{e}}$ siècle, le Maghreb a représenté, dans le domaine archéologique, le plus grand champ d'action que la France ait jamais possédé : cela ne semble pas un hasard si, à l'intérieur de ce groupe antiquisant, la "corporation " la plus représentée est celle des "Romains ». Ces anciens membres de l'École française de Rome ont été régulièrement envoyés en Algérie, en Tunisie, plus rarement au Maroc, pour faire leurs premières armes sur le terrain archéologique. On distingue de surcroît trois "Athéniens ", Charles Picard, dont la carrière s'achève dans ces années-là, Pierre Demargne et André Boulanger.

On voit mal comment la spécialité des antiquisants impliqués dans cette déclaration du 23 mai aurait pu les préparer à une compréhension pénétrante des colonies et de leur devenir. Du moins, aucun ne semble avoir choisi d'aborder des sujets antiques pour mieux réfléchir au présent colonial. Et personne, ici, n’est africaniste à proprement parler. Il y a ceux qui se sont consacrés à la littérature ancienne, tels Perret (1906-1992), traducteur de Virgile, ou Boulanger (1886-1958) qui, après sa thèse sur Ælius Aristide ${ }^{8}$, a pris la suite de Louis Gernet, pour la partie hellénistique du Génie grec dans la religion (1932), avant de traduire plusieurs discours de Cicéron. Pierre Demargne (1903-2000) est spécialiste d'archéologie grecque et Jacques Heurgon (1903-1995) étruscologue. Les travaux de Pierre Boyancé (1900-1976) sur la philosophie antique ${ }^{9}$, de William Seston (1900-1983) sur la Tétrarchie ${ }^{10}$ et de Pierre Wuilleumier (1904-1979) sur la cité de Tarente ${ }^{11}$ ne paraissent pas non plus avoir été les propédeutiques idéales à une prise de position sur le cas algérien. Un seul en vérité, Jean Bérard (1908-1957), fils de l'illustre Victor, s'est intéressé à certaines colonies de l'Antiquité, les fondations grecques d'Italie du Sud et de Sicile ${ }^{12}$.

Au sein de ses antiquisants s'exprimant en faveur de l'Algérie française, Charles Picard (1883-1965) peut incarner la figure du maître. Élève puis directeur de l'École

8 A. Boulanger, Alius Aristide et la sophistique dans la province d'Asie du II siècle de notre ère, Paris, De Boccard, 1923, XIV-504 p.

9 P. BoyancÉ, Le Culte des Muses chez les philosophes grecs. Études d'histoire et de psychologie religieuses, Paris, De Boccard, 1937, 375 p.

10 W. Seston, Dioclétien et la tétrarchie, Paris, De Boccard, 1946, 398 p.

11 P. Wuilleumier, Tarente, des origines à la conquête romaine, Paris, De Boccard, 1939, VI-752 p.

12 J. BÉrARD, La colonisation grecque de l'Italie méridionale et de la Sicile dans l'antiquité : l'histoire et la légende, Paris, De Boccard, 1941, 544 p. 
française d'Athènes, auteur d'une thèse monumentale sur Éphèse et Claros ${ }^{13}$, il a été un archéologue actif à Délos et Mallia. Ancien combattant en 1914-1918, ayant fait preuve d'une " attitude résistante ${ }^{14}$ " pendant la Seconde Guerre mondiale, refusant d'être recteur à la Libération, c'est politiquement un conservateur. Picard a été de plus inspecteur de l'archéologie marocaine. Il a formé beaucoup de générations d'antiquisants. Mais dans la liste des signataires, la présence du commandeur ne suffit pas à expliquer l'engagement de ses cadets. L'idée que les plus jeunes ont emboîté les pas du maître doit être partiellement révisée ${ }^{15}$.

\section{Les raisons d'un engagement}

Au vrai, chacun est arrivé par des canaux différents à la décision de soutenir l'Algérie française. Tout d'abord, par une forme d'" enracinement " : ainsi de Jacques Heurgon, qui est le seul à avoir vécu longtemps à Alger parmi les "Romains " dont les noms s'alignent dans cette déclaration du 23 mai 1956. Heurgon a non seulement consacré un article à Tipaza ${ }^{16}$ dans ses jeunes années, mais il a aussi enseigné en Algérie. C'est à l'automne 1932 qu'il a été nommé, en qualité de " chargé de cours ", à la faculté d'Alger. Un de ses étudiants s'appelle Albert Camus, qui lui dédie en 1939 ses Noces à Tipasa. Heurgon reste une dizaine d'années à son poste algérois, sa première affectation universitaire. Il y rédige sa thèse sur Capoue ${ }^{17}$. Sa correspondance avec Jean Tardieu révèle une absence de prise en compte des ferments de la crise algérienne ${ }^{18}$. Mais le recours à une source unique, cet échange de lettres avec

13 Ch. PICARD, Éphèse et Claros : recherches sur les cultes et sanctuaires de l'Ionie du Nord, Paris, De Boccard, 1922, XLVI-786 p.

14 C. ChARLE, "Charles Picard", dans Dictionnaire biographique des universitaires aux XIX et XXe siècle, vol. II. Les professeurs de la faculté des lettres de Paris (1909-1939), Paris, Éditions du CNRS, 1986, p. 172.

15 J.-Fr. SiRinelLI, " Guerre d'Algérie, guerre des pétitions ? Quelques jalons », Revue historique, 112/1 (1988), p. 73-100. Et plus exactement n. 47, p. 88. J.D. Le SuEUR n'analyse pas cette déclaration du 23 mai dans son Uncivil War. Intellectuals and Identity Politics During the Decolonization of Algeria, Philadelphia, University of Pennsylvania Press, 2001, X-342 p.

16 J. Heurgon, "Nouvelles recherches à Tipasa, ville de la Maurétanie Césarienne ", Mélanges d'archéologie et d'histoire 47 (1930), p. 182-201.

17 ID., Recherches sur l'histoire, la religion et la civilisation de Capoue préromaine, des origines à la deuxième guerre punique, Paris, De Boccard, 1942, XIV-483 p.

18 Voici par exemple une lettre d'Heurgon, datée du 27 octobre 1935, qui laisse apercevoir certaines difficultés rencontrées par le jeune professeur et son épouse : "Nous nous sommes réinstallés ici sans enthousiasme, et avons pourtant succombé à notre illusion annuelle, qui consiste à se dire : cette fois-ci, ce ne sera pas comme les années précédentes. Nous sommes revenus, Anne et moi, avec l'intention de vivre très repliés sur nous-mêmes, de ne consacrer que le minimum indispensable à nos connaissances algéroises, de ne pas 
Tardieu, qui est poète, déforme quelque peu notre vision du rapport d'Heurgon à l'Algérie. En effet, la correspondance Heurgon/Tardieu verse souvent dans l'intimité de chacun et leur amour commun de la littérature. On trouve rarement une note sur leur positionnement politique : en 1936, à l'occasion de la visite à Alger d'André Gide, un groupe d'étudiants nommé " Groupe d'Études Sociales ", demande à Heurgon de prononcer une conférence sur l'écrivain. L’intéressé traduit son malaise dans une nouvelle lettre : "Ce groupe est communisant; je ne le suis pas. Comment apprécier sincèrement le communisme de Gide (qui d'ailleurs est très noble et très humain), sans le trahir publiquement ${ }^{19}$ ? " Jacques Heurgon, fils de grands joailliers de la rue Royale, n'est assurément pas un homme de gauche, ce qui lui vaut quelques troubles familiaux quand son fils, Marc, crée en 1960 le PSU, parti socialiste unifié, constitué autour du soutien à l'indépendance algérienne ${ }^{20}$. Heurgon est de cette fraction d'enseignants de la faculté d'Alger qui attend son retour en métropole ${ }^{21}$. Il l'obtient à la Libération, après avoir participé à la campagne d'Italie. Par la suite, il maintient longtemps son point de vue sur l'Algérie coloniale.

Tout comme William Seston (1900-1983), qui signe lui aussi cette déclaration du 23 mai. La réaction est, chez ce dernier, " épidermique ", dirigée contre Marrou. Dix auparavant, au sortir de la guerre, l'animosité de Seston à l'égard de Marrou était déjà connue, Jacques Le Goff - relatant ses souvenirs d'étudiant à la Sorbonne - s'en est fait l'écho, par l'anecdote d'une « conspiration » bibliographique conduite par Seston

chercher ici d'impossibles amitiés (alors que nous en avons de sincères en France), de ne pas aller au cinéma voir de mauvais films doublés, etc. [...] Nous commençons à nous sentir sur la pente déclinante. Et à trouver notre vie ici bien austère [...] J'ai beaucoup travaillé, profitant de ce que les cours n'ont pas encore recommencé mais cette thèse est un pensum bien aride. Au fond c'est peut-être elle qui a contribué à me faire prendre Alger en grippe " (J. HeURGON et J. TARDIEU, Le ciel a eu le temps de changer. Correspondance 1922-1944, Paris, Mimec, 2004, p. 187-188).

19 Ibid., p. 192 (lettre du 30 mars 1936).

20 Marc Heurgon (1926-2001), auteur d'une Histoire du PSU (tome I, La fondation et la guerre d'Algérie, Paris, La Découverte, 1994, 444 p.).

21 Avant de devenir l'assistant de Pierre Bourdieu, Abdelmalek Sayad (1933-1978) a été étudiant en psychologie à Alger au début des années 1950, au cours desquelles il a remarqué une division entre deux " camps » universitaires : "Les professeurs se partageaient en gros en deux clans avec des pouvoirs séparés : un clan qui opte pour le pouvoir intellectuel et un autre qui louche vers le pouvoir plutôt politique. Les premiers, plus universitaires, regardaient plutôt vers Paris, vers la Sorbonne dont ils attendent la consécration. Les seconds, plutôt des bâtards de l'intellectualité et de l'Université malgré leurs titres, donnaient l'impression de n'être là, dans le pôle universitaire, que par un fâcheux compromis : ils penchent du côté du pouvoir politique ou administratif, du côté du gouvernement général et, au fond, du côté de l'ordre colonial tel qu'il est compris et vécu à Alger (pas même tel qu'on se le représente à Paris) " (cité par Pierre Bourdieu, Esquisses algériennes, Paris, Seuil, 2008, p. 27). 
à l'encontre de Marrou ${ }^{22}$. Même si leurs années de formation se ressemblent (École Normale, École de Rome), même s'ils se sont partagés un même domaine de recherche (la fin de l'Antiquité), les deux hommes sont irréconciliables. D'autant qu'il existe, entre eux, un fossé confessionnel ${ }^{23}$ : Marrou est un catholique convaincu, son adversaire est réformé. En rejoignant les rangs des partisans de l'Algérie française, Seston suit son coreligionnaire Soustelle ${ }^{24}$. Pourtant, le conseil de la Fédération protestante de France (FPF) effectue une protestation solennelle, le 12 mars 1957, renouvelée le 23 mars 1958, adjurant « les pouvoirs publics de mettre un terme aux agissements qui portent à la France un préjudice incalculable ${ }^{25} \%$. Ce qui ne suffit pas à faire changer d'avis Seston, qui reçoit au début du conflit, par son élève Marcel Le Glay, des nouvelles inquiétantes d'Algérie ${ }^{26}$. Il finit par signer aux côtés d'Heurgon, le "manifeste des intellectuels français ", en octobre 1960, un mois avant qu’à Montbéliard la FPF déclare que « la poursuite de la guerre accélère inexorablement la détérioration morale

22 "C'est dans un exposé bibliographique qu[e Seston] disait : "En ce qui concerne l'histoire de l'éducation, il y a un livre qui s'impose, c'est la Paideia de Jäger. Si vous ne lisez pas l'allemand, vous pouvez voir l'adaptation que M. Marrou en a donnée en français." Il faut dire que Seston détestait Marrou : c'était le protestant en face du catholique et puis, à la fin de sa vie, leur évolution politique a rendu leurs rapports encore plus difficiles. Seston s'est tourné vers l'extrême droite, il est devenu terriblement "Algérie française" " (J. LE Goff, Entretiens avec Marc Heurgon. Une vie pour l'histoire, Paris, La Découverte, 1996, p. 45).

23 Ils ont, de surcroît, deux conceptions divergentes du métier d'historien : tout au long de sa carrière, Marrou multiplie ses réflexions sur l'actualité et en fait part à ses étudiants, alors que Seston préfere camper sur son statut d'érudit.

24 Cf. supra, note 4.

25 Cf. Ph. WolfF, Les protestants en France. 1800-2000, Toulouse, Privat, 2001, p. $155-156$.

26 Bibliothèque du centre Gernet-Glotz (Paris), fonds Seston, lettre de Marcel Le Glay (Alger, le 15 novembre 1955) : "J'ai bien reçu vos deux dernières lettres. Si je n'y ai pas répondu plus tôt, c'est que nous venons, M. Lassus et moi, de faire un petit séjour à Djemila. Melle Allais comprend très bien que vous ne veniez pas en ce moment, alors que des bandes insurgées, étrangères à la région, commencent à sévir dans la commune mixte de Fedj-Mzala, surtout naturellement dans la zone montagneuse. On en est à la phase des enlèvements de personnes, des réquisitions et des marques d'infamie à l'encontre de musulmans pro-français, à qui on coupe les nez en particulier. Tout cela n'est pas drôle - Pour la première fois je suis allé à Djemila par le train ; le long des voies ce ne sont que poteaux coupés. Bref, si la situation ne s'améliore pas dans l'Aurès, où le général Parlange semble fort bien réussir, elle empire partout ailleurs. Nous allons donc orienter les recherches vers l'Oranie et faire un gros effort de publication. " Les archives de William Seston sont conservées par Rosine Adda, bibliothécaire attachée au centre ANHIMA-UMR 8210. Je remercie Michel Christol de m'avoir autorisée à consulter ce fonds Seston. 
et juridique de l'État ${ }^{27}$ ». Seston illustre donc les profondes tensions qui parcourent le protestantisme français à cette époque ${ }^{28}$.

Jean Bérard, quant à lui, forme un cas singulier. Il est le seul à avoir été directement préparé par son sujet de thèse à la mise en perspective des processus coloniaux dans la longue durée. Par ailleurs, de tous les professeurs qui signent cette déclaration du 23 mai, Bérard est certainement le plus à gauche. Les questions politiques l'attirent depuis longtemps. Dans les années 1930, à l'imitation de son propre père qui à la fin du XIXe siècle n'avait pas hésité à prendre sa plume pour traiter des problèmes contemporains ${ }^{29}$, Jean Bérard a écrit, sous les initiales C.M.R., une histoire de l'Italie mussolinienne très documentée, publiée en $1938^{30}$. Autre marque de cet engagement politique : Bérard a été, sous le fascisme, le correspondant d'un archéologue italien aux convictions "méridionalistes ", Umberto Zanotti Bianco ${ }^{31}$. Il aurait été intéressant d'observer l'évolution de la conscience algérienne de Bérard dans le cours du conflit, mais il meurt dans un accident de voiture en 1957. Un article paru à titre posthume dans La Revue historique, intitulé "L'homme fait-il son histoire 32 ? ", indique que son auteur se tenait éloigné du marxisme. Comme une partie de ses contemporains dont la sensibilité politique se situe à gauche, il peut avoir placé sa confiance dans le gouvernement socialiste de Guy Mollet, formé au début de l'année.

Pierre Wuilleumier et Pierre Boyancé, pour leur part, ne sont en rien des « africains ». Comme « jeune romain » envoyé en Algérie, Wuilleumier se voit confier l'étude d'objets en bronze du Musée d'Alger, notamment une série de trépieds : il caractérise les rivages numides comme " des lieux trop barbares pour les avoir produits ${ }^{33}$ " et préfere bien vite retourner en Italie. Son passage par l'Algérie ne parait pas l'avoir durablement marqué. À la suite de cette courte excursion africaine, lorsqu'il s'agit de désigner les "stagiaires " qui vont le relayer, il signifie sa préférence pour son camarade Boyancé, attaché comme lui à l'histoire de la religion romaine, plutôt que pour

27 WolfF, Les protestants en France. 1800-2000, p. 156.

28 G. Pervillé, "Les protestants et la guerre d'Algérie », Bulletin de la Société de l'histoire du protestantisme français 150 (octobre-novembre-décembre 2004), p. 683-701.

29 À la fin du XIX ${ }^{e}$ siècle, Victor Bérard (1864-1931) publie une série de textes sur l'actualité grecque et turque : La Turquie et l'hellénisme contemporain (1893), La politique du sultan (1897), La Macédoine (1897), Les affaires de Crète (1898).

30 C. M. R (J. BÉrard), Histoire du fascisme italien (1919-1937), Paris, Les Éditions Rieder, 1938, 293 p.

31 Une partie de la correspondance entre Jean Bérard et Umberto Zanotti Bianco (18891963) est conservée par la section romaine de l'ANIMI (Associazione nazionale per gli interessi del Mezzogiorno italiano). Cf. aussi N. DE HAAN, «Umberto Zanotti Bianco and the Archaeology of Magna Grecia during the Fascist Era ", Fragmenta (Journal of the Royal Netherlands Institute in Rome) 2 (2008), p. 233-250.

32 J. BÉrARD, "L'homme fait-il son histoire? ", Revue historique 218 (1957), p. 241-260.

33 P. Wuilleumier, "Mobilier de l'Afrique romaine ", Mélanges d'archéologie et d'histoire 45 (1928), p. 149. 
Heurgon ${ }^{34}$. En définitive, c'est Heurgon qui est mandaté outre-mer et Boyancé n'écrit aucun article africain.

D'un savant à l'autre, l'Algérie française est donc défendue pour des motifs différents : peut-être en adhésion au socialisme du gouvernement en place (comme Bérard, semble-t-il) ; à la suite d'une carrière africaine (c'est la situation d'Heurgon); et, bien sûr, pour ne pas laisser sans suite l'audace de Marrou, qui - comme manière de plusvalue à son " J'accuse " - a commis l'affront de se placer le premier sous la bannière de la «Sorbonne».

Cette apologie de l'Algérie française a-t-elle été préparée par une certaine manière d'écrire l'histoire antique de l'Afrique du Nord ? L'exercice d'une discipline (les études classiques) peut-il asseoir une réflexion politique?

\section{Les antiquisants et l'Algérie antique}

Peu de savants se sont penchés sur le cas de l'ancienne Africa avec des arrière-pensées nettement " colonialistes ». À la fin du XIX siècle, René Cagnat a parfois mis en parallèle la conquête romaine et la présence française outre-Méditerranée dans son ouvrage sur L'armée romaine en Afrique (1892), mais sans faire de cette comparaison un usage explicatif systématique. Jules Toutain (1865-1961) 35 est à coup sûr celui qui s'est le plus préoccupé de rapprocher la geste romaine dans la province africaine de Proconsulaire d'une part, et la colonisation française d'autre part, dans sa thèse sur Les cités romaines de Tunisie $(1896)^{36}$. Mais ces deux ouvrages datent encore des débuts de l'âge colonial. Par la suite, les travaux africains se font discrets sur le temps présent. Stéphane Gsell, détenteur de la chaire d'Histoire de l'Afrique du Nord au Collège de France, ne cède que rarement à la tentation d'une analogie explicite entre l'Antiquité et l'époque dont il est le contemporain, lors de sa leçon inaugurale en $1912^{37}$.

L'histoire africaine paraît, somme toute, peu instrumentalisée, tout comme l'archéologie. Du XIX ${ }^{\mathrm{e}}$ au XXe siècle, le choix des sites archéologiques fouillés au Maghreb par les membres de l'École de Rome répond à un seul grand critère : il faut avant tout chercher « du " romain, avant «le " punique, «le " numide, «le " byzantin. Et conduire un chantier bien circonscrit : une basilique, un quartier d'habitations, un tronçon d'aqueduc, un praetorium. On collecte en outre les inscriptions. On

34 Bibliothèque de l'Institut de France, fonds Carcopino, lettre de Pierre Wuilleumier à Jérôme Carcopino (29 mars 1929).

35 Cf. dans ce dossier, la communication d'Aurélie Rodes, p. 59-69.

36 J. TOUTAIN, Les cités romaines de la Tunisie. Essai sur l'histoire de la colonisation romaine dans l'Afrique du Nord, Paris, Fontemoing, 1896, 412 p.

37 S. Gsell, Histoire de l'Afrique du Nord. Leçon d'ouverture de la chaire d'histoire de l'Afrique du Nord au Collège de France, Paris, Éditions de la Revue politique et littéraire et de la Revue scientifique, 1912, 34 p. 
encourage de même à s'intéresser aux édifices chrétiens, non pour favoriser l'héritage religieux occidental, mais parce que fouiller une église n'implique pas de grandes dépenses ${ }^{38}$.

Dans la discussion sur les problèmes rencontrés par les Romains en Afrique du Nord, certains ont été conduits à mentionner le rôle des " ethnies " africaines. Les Berbères deviennent alors l'objet de beaucoup d'interrogations. Lors de son séjour dans l'oasis d'El-Kantara, Marrou évoque de la sorte " le destin tragique de la race berbère qui n’a jamais eu ni unité politique, ni langue de culture, ni civilisation à elle $^{39}$ ». Liées au " problème " berbère, les données de la géographie ${ }^{40}$ soutiennent d'autres mises en perspective de la "romanisation ». On définit donc deux Afriques, celle des montagnes et celle des plaines, préfigurant des enquêtes réalisées plus tard par Christian Courtois ${ }^{41}$ et par Philippe Leveau dans l'arrière-pays de Cherchell ${ }^{42}$. Déjà, au début du Xx siècle, Stéphane Gsell et Henri Graillot, partant des données épigraphiques de Timgad et Khenchala, cherchent à expliquer les nombreuses erreurs des lapicides par un "défaut d'assimilation " dû au " voisinage de l'Aurès ${ }^{43}$ ". Ce terme d' "assimilation " revient souvent dans les débats sur la "romanisation " de l'Afrique, au gré de divers dossiers, concernant l'onomastique, l'adoption des cultes romains, les équipements urbains. Les débats coloniaux du temps présent imprègnent le vocabulaire des érudits. D'une publication à l'autre, nombreux sont ceux qui cherchent à évaluer le degré d'" assimilation " des anciens Africains. Dans un article de jeunesse, fondé sur les découvertes épigraphiques de Rapidum, William Seston passe ainsi en revue les noms rencontrés dans les épitaphes et conclut que «l'assimilation des Berbères fut d'autant plus rapide qu'une étroite communauté d'intérêts lia de bonne heure indigènes et Romains ${ }^{44}$ ". À propos de Gilbert Picard, le fils de

38 Cf. Bibliothèque de l'Institut de France, fonds Carcopino, lettre de Stéphane Gsell à Jérôme Carcopino (Paris, 7 août, probablement 1912, quand Carcopino est à Alger) : " [fouiller] une église, ça ne coûte pas cher. » Gsell est nommé inspecteur des Antiquités de l'Algérie en 1900.

39 H.-I. Marrou, "La collection Gaston de Vulpillières à El-Kantara ", Mélanges d'archéologie et d'histoire 50 (1933), p. 85-86 : "Ce n'est pas le moindre intérêt de la collection de Vulpillières que de nous apporter quelques lumières sur un des points les moins connus de l'histoire religieuse du pays berbère. "

40 La géographie vidalienne constitue, pour tous ces historiens, une référence constante.

41 C. Courtois, Les Vandales et l'Afrique, Paris, Arts et métiers graphiques, 1955, 457 p. Courtois évoque à plusieurs reprises les « difficultés " géographiques de l'Afrique, qu'il conçoit comme une " malédiction" (n. 99, p. 299).

42 Ph. Leveau, Caesarea de Maurétanie : une ville romaine et ses campagnes, Paris, De Boccard, 1984, IX-556 p.

43 S. Gsell et H. Graillot, "Ruines romaines au nord de l'Aurès ", Mélanges d'archéologie et d'histoire, 13 (1893), p. 460-541.

44 W. SESTON, "Le secteur de Rapidum sur le Limes de Maurétanie césarienne après les fouilles de 1927 », Mélanges d'archéologie et d'histoire 45 (1928), p. 173. 
Charles, et de sa Civilisation de l'Afrique romaine (1959), André Mandouze notera plus tard que cet " ouvrage est dans son ensemble assez représentatif de la thèse de l'“assimilation”, Rome étant présentée comme une magistra vitae suivie et adoptée par une Afrique tout à la fois docile et originale ${ }^{45}$ ». Mais la devise du PPA (Parti du peuple algérien) fondé en 1937 par Messali est précisément « ni assimilation, ni séparation, mais émancipation ». Et dans son Journal, Mouloud Feraoun note : "Non : l'assimilation, fût-elle sincèrement désirée, n'est pas viable ! Les cartes dans ce jeu sont truquées. Tous ceux que j'ai rencontrés savaient que je n'étais ni Français, ni intégrable ${ }^{46}$. " Progressivement, il devient embarrassant de parler d'assimilation, le mot s'efface des publications d'histoire ancienne.

Sous la plume de ces antiquisants, le recours fréquent à un autre terme, plus exactement une autre expression - " terre d'Afrique ${ }^{47}$ " - ne constitue-t-il pas une manière de retirer à l'espace algérien sa spécificité ? Cette évidente allusion à l'Africa des Anciens paraît à la fois pompeuse, désuète et condescendante. Or c'est peutêtre par l'angle topographique et géographique de leurs enquêtes que ces historiens auraient pu discerner l'originalité du territoire algérien, autrefois romain. De proche en proche, certains se seraient interrogés sur l'économie et la société de l'Algérie. Mais parmi ceux qui signent cette déclaration du 23 mai aucun n'a, par exemple, étudié le latifundium et l'esclavage antiques, personne non plus ne s'est penché très longtemps sur le cas des donatistes, schismatiques dont les revendications sont aussi sociales, et personne n'a suivi de près le sort de ceux auxquels on les rattache souvent, les circoncellions ${ }^{48}$.

Chez ces savants qui ont traversé la Méditerranée pour compléter leur formation ou mener une carrière, la figure de Firmus, rebelle contre Rome à la fin du IV siècle, n'a

45 A. Mandouze, Saint Augustin. L'aventure de la raison et de la grâce, Paris, Études augustiniennes, 1968, n. 1, p. 339.

46 Cité par B. STORA, Les mots de la guerre d'Algérie, Toulouse, PUM, 2005, p. 15, s. v. " assimilation ".

47 Citons par exemple le discours d'Aimé Puech (1860-1940), spécialiste de littérature ancienne, qui évoque "les terres africaines » ou la recension d'un ouvrage de Gilbert Picard, par Raymond Thouvenot (1896-1981), autre élève de l'École de Rome : «Le livre de M.G. Picard, si riche et si alerte, sera lu avec reconnaissance par tous ceux qui, après avoir vécu sur cette terre d'Afrique, continuent à l'aimer " (Journal des Savants, 1960, p. 139).

48 À la différence de Charles Saumagne (1890-1972), auteur d'un article, court mais important sur cette question, lue à travers le prisme marxiste. Ce travail parait dans les Annales de Lucien Febvre et Marc Bloch : "Ouvriers agricoles ou rôdeurs de celliers ? Les circoncellions d'Afrique ", Annales d'histoire économique et sociale, 1934, 6, p. 355-364. Avocat, historien, archéologue, Saumagne a été un acteur de l'indépendance tunisienne. Cf. C. Gutron, L'archéologie en Tunisie. XIX'-XXe siècles. Jeux généalogiques sur l'Antiquité, Paris-Tunis, Karthala, 2010, 327 p. 
pas non plus fait l'objet de beaucoup de soins ${ }^{49}$. Parce qu'elle appartient à une " antiquité tardive » longtemps négligée, ou parce que les aventures du Maure ont laissé peu de traces matérielles, ou enfin parce qu'on préfere souligner les moments d'unanimité autour d'une romanité jugée volontiers civilisatrice.

\section{Jean Bérard, une exception?}

Jean Bérard est justement l'un des rares à prouver que ce domaine économique et social l'intéresse. Parti comme jeune "stagiaire " de l'École de Rome en Algérie, il a étudié des mosaïques de Cherchell (Caesarea) représentant divers travaux agricoles ${ }^{50}$. À cette occasion on le voit accorder une attention particulière à l'outillage des paysans africains. Il s'attarde sur les techniques de labour représentées dans ce cycle de mosaïques. Il note qu' " à Cherchel, on peut voir les indigènes labourer encore avec des araires de bois en tous points comparables à la charrue romaine de la mosaïque ${ }^{51}$ " et observe, entre les deux modèles, les ressemblances dans le timon renforcé par une ligature et le soc de fer. Il analyse ensuite les points de convergence entre les modes de culture antiques et le dry-farming moderne. Il scrute les gestes du semeur puis décrit les façons anciennes de tailler la vigne. Bref, il produit une étude encore " antiquaire ", dont on sent qu'elle pourrait devenir une première histoire sociale.

Mais ici, Bérard est loin de son sujet sur les apoikiai grecques, auxquelles il consacre un ouvrage paru en $1941^{52}$. Il ne faut pas croire, au demeurant, qu'il ait choisi ce sujet pour méditer sur les créations coloniales à travers les siècles. C'est en vérité sa piété filiale qui lui dicte cette recherche : son père avait, en son temps, déjà tenté de montrer tout le profit qu'il y avait à tirer des récits légendaires, dans l'histoire archaïque de la Méditerranée ${ }^{53}$. Son fils a le même cheval de bataille. Qu'il décrive les fondations de Sélinonte ou de Mégara, de Cumes ou Syracuse, Bérard ne superpose pas les registres antiques et contemporains. Il ne fait pas preuve de compassion pour les indigènes quand il voit les colons grecs employer la manière forte : ainsi, il raconte sans broncher comment les Métapontins se battent pour s'emparer des champs fertiles des Oenôtres ${ }^{54}$. S'il ne s'attriste pas des incidents provoqués par

49 Cf. Y. Modéran, « Gildon, les Maures et l'Afrique ", MEFRA 101 (1989), p. 821-872.

50 J. BÉrARD, "Mosaïques inédites de Cherchel ", Mélanges d'archéologie et d'histoire 52 (1935), p. 113-142.

51 BÉrard, " Mosaïques inédites de Cherchel », p. 133.

52 Voir supra, n. 12.

53 Notamment dans Les Phéniciens et l'Odyssée (1902-1903, 1927²) et Les navigations d'Ulysse (1925-1927).

54 J. BÉRARD, La colonisation grecque de l'Italie méridionale et de la Sicile, p. 199 : «L'ambition des Métapontins fut de s'assurer la possession des terres à blé qui l'entouraient de toutes parts. Pour les conquérir, dit Antiochos, les Achéens durent faire la guerre aux Oenôtres 
les nouveaux venus, c'est aussi parce qu'il croit, avec Denys d'Halicarnasse, qu'avant même le mouvement colonisateur, les habitants de l'Italie sont déjà des Grecs comme les autres 55 . Tardivement créée, la colonie de Thourioi, la seule peut-être à pouvoir être comparée à une colonie contemporaine par son versant "idéologique ", ne l'intéresse pas particulièrement ${ }^{56}$. Bref, Bérard ne confond jamais en esprit les colonisations ancienne et moderne, reconnaissant qu'il s'agit de phénomènes bien distincts.

Les questionnaires en faveur parmi les "Romains " qui ont travaillé en Afrique (et en Italie) n'ont donc pas préparé le terrain d'une réflexion originale sur la valeur, les objectifs, les dangers de l'entreprise coloniale. Ces historiens n'ont pas voulu mélanger les genres : positivistes, ils s'en sont tenus à des études aussi strictement informatives que possibles, ne laissant pas percer leurs éventuelles préoccupations des problèmes de l'heure.

\section{Conclusion}

Que retenir de cette rapide analyse?

Pour la plupart, les antiquisants qui ont signé la déclaration du 23 mai 1956 sont conservateurs, à l'exception du cas singulier de Jean Bérard. En 1960, face au manifeste des 121 qui encourage à l'insoumission, Heurgon devient un des fers de lance du « Manifeste des intellectuels français » que signera aussi William Seston. Et quand les Écoles Françaises à l'étranger sont vilipendées en 1970 dans un article du Monde,

qui habitaient l'arrière-pays, ainsi qu'aux Tarentins. " D'après un fragment d'Antiochos, apud Strabon, VI, 265 ( $F H G$ I, p. 184 fr B).

55 Ibid., p. 492 : «À cette doctrine panpélasgique résultant des données traditionnelles, ne convient-il pas d'opposer un certain scepticisme ? On y serait d'autant plus enclin que l'objet de Denys d'Halicarnasse dans ses Antiquités romaines est de démontrer l'origine grecque des populations italiennes, et que cette thèse se retrouve dans d'autres auteurs latins tels que Justin. Cette croyance à l'origine pélasgique des plus anciennes populations de l'Italie méridionale ne serait-elle pas une invention tardive dépourvue de tout fondement, ou le fruit de déductions savantes ? On pourrait fort bien se poser la question, si ces données n'étaient complétées par les traditions concernant les Pélasges d'Étrurie ou les Étrusques eux-mêmes, et si ces traditions ne semblaient trouver confirmation dans les découvertes archéologiques et les investigations de la linguistique. "

56 À propos de Thourioi, Bérard écrit : « La ville fut construite sur un plan régulier, conformément aux idées d'urbanisme d'Hippodamos de Milet, qui vint y habiter ; et les citoyens selon leur origine furent divisés en dix tribus [...] La fortune de Thurioi fut plus brève encore que celle de l'ancienne Sybaris. Non seulement elle ne tarda pas à être divisée entre démocrates, partisans d'Athènes, et aristocrates, partisans de Sparte ; mais dès la fin $\mathrm{du} \mathrm{V}^{\mathrm{e}}$ siècle elle fut en butte aux assauts de plus en plus pressants des Lucaniens " (ibid., p. 164). 
intitulé "Citadelles et Mandarins 57 ", on ne s'étonne pas de voir Boyancé et Heurgon s'insurger contre cette attaque.

Les historiens qui sont montés au créneau pour défendre l'Algérie française en 1956 ne font guère preuve de prescience ; mais ils sont en accord, à cette date, avec la majorité de la population française, tenant à conserver la terre algérienne, considérée d'abord comme un département français.

Ces érudits promoteurs de "l'œuvre colonisatrice " n’ont que rarement mêlé à leurs travaux scientifiques les considérations sur la conquête française et ses suites. Dans la deuxième moitié du $\mathrm{xx}^{\mathrm{e}}$ siècle, ce sont plutôt les antiquisants anti-colonialistes, tel Yvon Thébert, qui ont rendu compte de leur choix politique au cour même d'études sur l'Antiquité africaine ${ }^{58}$.

À l'inverse de leurs aînées, les générations « romaines " des années 1950 seront porteuses d'idées pro-algériennes. Pendant la guerre, Paul Veyne, jeune historien de retour de Rome, côtoie ainsi les "porteurs de valise ${ }^{59}$ ", encouragé par son ami Georges Ville, lui aussi "romain ", auteur d'une thèse sur les gladiateurs ${ }^{60}$ et, ceci expliquant peut-être cela, prosélyte de l'engagement physique. Leur condisciple de l'EFR, Paul-Albert Février, chrétien libéral, attaché à la figure de Marrou, est acquis lui aussi à la cause algérienne ${ }^{61}$.

Dans son ouvrage Les Guerres sans fin, Benjamin Stora a montré les mécanismes par lesquels une certaine mémoire algérienne a nui à la compréhension rétrospective de la guerre : Stora décrit « le mythe de l'Algérie française heureuse [...] renforcé par la nostalgie, les souvenirs, cette sorte de passé embelli ${ }^{62}$. "Par ailleurs, dans un essai d'ego-histoire, Raoul Girardet a désigné l'Université française d'avant la crise de 1968 comme " le temps de la Sorbonne heureuse ${ }^{63}$ ". En définitive, l'étude des problèmes soulevés par la déclaration du 23 mai 1956 illustre, sous certains aspects, la réunion d'états de grâce supposés, la conjonction de deux bonheurs qui ne voulaient pas être entamés par les à-coup de l'actualité, l'association de deux "mythes ", celui de

57 M. Denuzière, "Citadelles et Mandarins ", Le Monde, 5 août 1970.

58 Y. THÉBERT, "Romanisation et déromanisation en Afrique : histoire décolonisée ou histoire inversée?", Annales. Économies, sociétés, civilisations 33 (1978), p. 64-82.

59 Cf. P. Veyne, Le quotidien et l'intéressant. Entretiens avec Catherine Darbo-Peschanski, Paris, Les Belles Lettres, 1995, p. 29-35. Sur les positions de Pierre Vidal-Naquet, cf. ici même, l'article de Pauline Schmitt-Pantel.

60 G. VIlle, La gladiature en Occident, des origines à la mort de Domitien, Rome, EFR, 1981, X-519p.

61 J.-M. Guillon (dir.), Paul-Albert Février, un historien dans l'Algérie en guerre : un engagement chrétien. 1959-1962, Paris, Cerf, 2006, 525 p.

62 Et, de fait, la plupart des anciens «Romains " qui signent cette pétition coloniale ont connu l'Algérie vingt ou trente ans auparavant : consciemment ou non, ils peuvent exprimer une forme de regret du temps passé.

63 R. GiRARDET dans Essais d'ego-histoire, réunis et présentés par Pierre Nora, Paris, Gallimard, 1987, p. 151, qui compare aussi la Sorbonne d'alors à une "République patricienne". 
"l'Algérie heureuse ${ }^{64}$ " et celui de la "Sorbonne heureuse ": deux prétendus âges d'or qui, du malheur indigène, n'ont pas su faire cas.

Sarah REY

PLH-ERASME

Université de Toulouse (UTM)

Pavillon de la recherche

5, allées Antonio Machado

F-31058 Toulouse Cedex 9

sreylauzerte@yahoo.fr

64 B. Stora, Les guerres sans fin. Un historien, la France et l'Algérie, Paris, Stock, 2008, p. 24. 